\title{
The genetics of breast cancer: risk factors for disease
}

This article was published in the following Dove Press journal:

The Application of Clinical Genetics

6 January 2011

Number of times this article has been viewed

\section{Andrew Collins Ioannis Politopoulos \\ Genetic Epidemiology and Bioinformatics Research Group, Human Genetics Research Division, Southampton General Hospital, School of Medicine, University of Southampton, Southampton, UK}

Correspondence: Andrew Collins Genetic Epidemiology and Bioinformatics Research Group, Human Genetics Research Division, Southampton General Hospital, School of Medicine, University of Southampton, 808 Duthie Building, Tremona Rd, Southampton SOI6 6YD, UK Tel +442380796939

$\mathrm{Fax}+442380794264$

Email arc@soton.ac.uk
Abstract: The genetic factors known to be involved in breast cancer risk comprise about 30 genes. These include the high-penetrance early-onset breast cancer genes, BRCA1 and BRCA2, a number of rare cancer syndrome genes, and rare genes with more moderate penetrance. A larger group of common variants has more recently been identified through genome-wide association studies. Quite a number of these common variants are mapped to genomic regions without being firmly associated with specific genes. It is thought that most of these variants have gene regulatory functions, but their precise roles in disease susceptibility are not well understood. Common variants account for only a small percentage of the risk of disease because they have low penetrance. Collectively, the breast cancer genes identified to date contribute only $\sim 30 \%$ of the familial risk. Therefore, there is much interest in accounting for the missing heritability, and possible sources include loss of information through ignoring phenotype heterogeneity (disease subtypes have genetic differences), gene-gene and gene-environment interaction, and rarer forms of variation. Identification of these rarer variations in coding regions is now feasible and cost effective through exome sequencing, which has already identified high-penetrance variants for some rare diseases. Targeting more 'extreme' breast cancer phenotypes, particularly cases with early-onset disease, a strong family history (not accounted for by $B R C A$ mutations), and with specific tumor subtypes, provides a route to progress using next-generation sequencing methods.

Keywords: breast cancer, common and rare genetic variation, missing heritability, bioinformatics, exome sequencing

\section{Introduction}

Family history of breast cancer is known to be one of the strongest risk factors for this disease. For example, meta-analysis of familial breast cancer studies gives lifetime risk ratios of 1.80 in families with one affected first-degree relative, 2.93 in families with two affected relatives, and 3.90 in families with three affected relatives. ${ }^{1}$ Risk ratios are highest for cases at younger ages and, for a particular individual, are greater the younger their relative is diagnosed. The familial pattern of the disease provides clear evidence for the important role of genetic variation in determining risk. The identification of genetic factors involved in predisposition to breast cancer has been a topic of intensive study for more than 20 years. An important early breakthrough in the genetic dissection of the disease was linkage mapping, using breast cancer family data, of the $B R C A 1^{2}$ and $B R C A 2^{3}$ genes. Rare mutations in these genes confer high relative risks to carriers of 10 - to 20 -fold, corresponding to a $30 \%-60 \%$ risk by the age of 60 years, compared with $3 \%$ for the general population. ${ }^{4}$ These mutations account for $\sim 16 \%-20 \%$ 
of the familial risk of breast cancer in the general population. ${ }^{4,5}$ In addition, there are a number of rare to very rare highpenetrance gene variants that underlie cancer syndromes and a few rare genes that have more moderate penetrance. Collectively, the rare genes found to date account for $<25 \%$ of the familial risk. Recent studies have focused on the role of common genetic variation, through analysis of large samples of cases and controls tested for association at many thousands of single nucleotide polymorphism (SNP) markers. These studies have identified a number of common breast cancer genes and revealed new insights into the natural history of the disease. However, all these genes are low-penetrance variants that account for only a few percent of the familial risk. Because the bulk of the familial risk is unexplained by the genes identified thus far, research is focusing on identifying sources of the 'missing' heritability. This review considers what is known about the genetic basis of breast cancer and evaluates the clinical utility of the evidence, while emphasizing ongoing strategies to identify more of the genetic variation. New technologies, such as next-generation sequencing, and the development of novel bioinformatic approaches to analysis are at the forefront of this effort.

\section{Mendelian high-penetrance genes}

About 100 genes for genetic diseases showing Mendelian patterns of inheritance in families are known. ${ }^{6}$ These are invariably rare genes and associated with high relative risks. Most of the genes have been identified through linkage analysis of carefully selected families, followed by positional cloning. Within this category are the breast cancer $B R C A 1$ and BRCA2 genes, which contain over 1000 mutations. Genetic screening for the spectrum of important mutations in these genes in high-risk families is well established. The $B R C A 1$ 'breast cancer 1 early-onset' gene ${ }^{2}$ is involved in susceptibility to breast and ovarian cancer at a young age, and tumors can arise through somatic or germline mutations. Impaired or lost BRCA1 function underlies substantial genome instability including increases in the number of mutations, DNA breakage and chromatid exchanges, increased sensitivity to DNA damage, and defects in cellcycle checkpoint functions. The role of BRCA1 in the DNA damage response is that of 'caretaker' or 'master regulator' in the genome. ${ }^{7-9}$

Jensen et $\mathrm{al}^{10}$ isolated the large protein encoded by the $B R C A 2$ gene and showed it to be a key mediator of homologous recombination. It is a crucial element in the DNA repair process which, if impaired through mutation, can lead to chromosome instability and cancer. It is known to mediate recombinational DNA repair by promoting assembly of RAD51 onto single-stranded DNA. This has a key role in catalyzing the invasion and exchange of homologous DNA sequences. Mutations in the BRCA2 gene may disrupt this mechanism and impair repair of DNA breaks, using homologous sequences from an intact homolog or sister chromatid, leading to errors in the repair process and chromosome instability.

$B R C A 1$ and $B R C A 2$ are likely to be the only major high-penetrance genes underlying breast cancer. Germline mutations in the TP53 gene cause Li-Fraumeni syndrome, a phenotype which includes early-onset breast cancer, ${ }^{11}$ but these mutations are far rarer. Both $B R C A 1$ and $B R C A 2$ genes were identified using linkage mapping in families, a method that has been successful in identifying many Mendelian disease genes. However, this strategy has contributed little to the study of more common or 'complex' forms of disease, mediated by genetic variants with reduced penetrance which may interact with environmental and other genetic factors. The complexity of this pattern of inheritance greatly reduces the power to detect genes through family-based studies.

\section{Rare cancer syndromes and rare moderate-penetrance genes}

There are a number of syndromes that include breast cancer as a component of the disease phenotype. Rare to uncommon mutations in the $P T E N^{12}$ and $S T K 11^{13}$ genes cause Cowden and Peutz-Jeghers syndromes, respectively, and both are associated with considerably increased breast cancer risk. ${ }^{14}$ The E-cadherin gene $(C D H 1)$ encodes a cellular adhesion protein and is a powerful tumor suppressor of breast cancer. ${ }^{15}$ It is particularly implicated in invasive lobular breast carcinomas. $R A D 51 C$ is another gene involved in the recombinational repair of double-stranded DNA breaks. Rare germline mutations have been shown to confer increased risks of breast and ovarian cancer. ${ }^{16}$ Segregation in families follows Mendelian patterns, and the disease phenotype resembles that of BRCA1 and BRCA2 mutation carriers.

There are also a number of gene mutations associated with more moderate risks of breast cancer, which show marked departures from Mendelian patterns of inheritance. As a result, segregation of disease with the mutation may be unhelpful to confirm relationship with disease. Genes in this category include germline mutations in the ataxia-telangiectasia (ATM) gene, which are associated with increased risk ( $\sim 2.2$-fold) of breast cancer in carriers of heterozygous mutations, with apparently higher risks below the age of 50 years. ${ }^{17}$ Other rare moderate-penetrance genes include heterozygous mutations in $B R I P 1$ (encoding a $B R C A 1$-interacting protein) that 
confers elevated risks of breast cancer and Fanconi anemia subtype FA-J for bi-allelic mutations. The partner and localizer of BRCA2 (PALB2) gene interacts with BRCA2, and mono-allelic mutations are involved in familial breast cancer, conferring a 2.3-fold risk. Mutations in BRCA2 are also known to underlie Fanconi anemia (subtype FA-D1), and bi-allelic mutations of PALB2 underlie the very similar Fanconi anemia subtype FA-N. ${ }^{18}$ Rare variants in the cell cycle checkpoint kinase 2 (CHEK2) gene are known to underlie an approximately twofold increase in risk of breast cancer. Products of this gene are involved in DNA damage repair, and mutations are found in $1 \%-2 \%$ of unselected women with breast cancer. ${ }^{19}$

\section{Common low-penetrance breast cancer genes}

Genome-wide association studies (GWAS) use panels of up to a million or more SNPs to identify common gene variants in large case and control samples. GWAS have identified more than 100 such low-penetrance loci involved in cancer, including at least 17 related to breast cancer (Table 1).
These variants have allele frequencies in the range $0.05-0.5$, but they confer only small increases in disease risk. ${ }^{4}$ Because of the greatly reduced penetrance and strongly non-Mendelian patterns of inheritance, there is often considerable uncertainty about the exact underlying genetic mutation. Not only are the most strongly associated SNPs unlikely to be the causal sites (these are 'tags' selected to represent variation at many polymorphic sites that are not tested directly) but there also may be uncertainty about the gene involved. It has also been suggested that multiple rare variants create 'synthetic association' signals in a GWAS if they occur more often in association with a common tag SNP. This implies that causal variants could be many megabases away from variants detected in GWAS, ${ }^{20}$ although this scenario appears to be rare. ${ }^{21}$ Perhaps, one of the unexpected findings from these studies is a greater-thananticipated role for noncoding variants in common diseases. ${ }^{22}$ From the analysis of population sequences, ${ }^{23}<30 \%$ of common variants associated with disease are annotated as, or in linkage disequilibrium with, nonsynonymous (coding) variation. This supports the view that many of the common disease variants have gene regulatory roles.

Table I Known breast cancer susceptibility genes and regions

\begin{tabular}{|c|c|c|c|c|}
\hline Known gene/region & Location & Mapped by & Allele frequency & Known/possible function \\
\hline$B R C A I$ & |7q2| & Linkage & Rare & DNA repair/genome stability \\
\hline$B R C A 2$ & $13 q 13.1$ & Linkage & Rare & Recombinational repair \\
\hline TP53 & |7p|3.| & Linkage & Rare & Li-Fraumeni syndrome, apoptosis \\
\hline ATM & I Iq22.3 & Candidate resequencing & Rare & DNA repair \\
\hline$B R I P I$ & $17 q 23.2$ & Candidate resequencing & Rare & DNA repair, associated with $B R C A I$ \\
\hline CHEK2 & $22 q 12.1$ & Candidate resequencing & Rare & DNA repair/cell cycle \\
\hline PALB2 & $16 p 12.2$ & Candidate resequencing & Rare & Associated with $B R C A 2$ \\
\hline RAD5 / C & $17 q 22$ & Candidate resequencing & Rare & Homologous recombination repair \\
\hline PTEN & $10 q 23.3$ & Linkage & Rare & Cowden disease, cell signaling \\
\hline STKII (LKBI) & $19 p \mid 3.3$ & Linkage & Rare & Peutz-Jeghers syndrome, cell cycle arrest \\
\hline $\mathrm{CDHI}$ & $16 q 22.1$ & Linkage & Rare & Intercellular adhesion: lobular BC \\
\hline FGFR2 & $10 q 26$ & GWAS & Common & Fibroblast growth factor receptor \\
\hline TOX3 (TNRC9)/RBL2 & $16 q 12$ & GWAS & Common & Chromatin structure/cell cycle \\
\hline$M A P 3 K I$ & $5 q 11.2$ & GWAS & Common & Cellular response to growth factors \\
\hline LSPI & IIp|5.5 & GWAS & Common & Neutrophil motility \\
\hline $8 q 24$ & $8 q 24$ & GWAS & Common & Intergenic, enhancer of MYC proto-oncogene? \\
\hline $2 q 35$ & $2 q 35$ & GWAS & Common & \\
\hline CASP8 & $2 q 33$ & GWAS & Common & Apoptosis \\
\hline SLC4A7/NEKIO? & $3 p 24.1$ & GWAS & Common & Cell cycle control? \\
\hline COXI I/STXBP4? & $17 q 22$ & GWAS & Common & Transport? \\
\hline MRPS30? & $5 p 12$ & GWAS & Common & Apoptosis? \\
\hline NOTCH2/FCGRIB? & IpII.2 & GWAS & Common & Signaling/immune response? \\
\hline RAD5 ILI & 14q24.1 & GWAS & Common & Homologous recombination repair? \\
\hline CDKN2A/CDKN2B? & $9 p 21$ & GWAS & Common & Cyclin-dependent kinase inhibitors? \\
\hline MYEOV/CCNDL? & $1|q| 3$ & GWAS & Common & Cell cycle control/fibroblast growth factors? \\
\hline ZNF365? & $10 q 21.2$ & GWAS & Common & Zinc finger protein gene \\
\hline ANKRD / 6/FBXO/8? & $10 p \mid 5.1$ & GWAS & Common & Helicase? \\
\hline ZMIZI? & $10 q 22.3$ & GWAS & Common & Regulates transcription factors? \\
\hline
\end{tabular}

Notes: ? refers to 'possible' gene or function in the breast cancer context. There is uncertainty about the exact genes and their functional roles in breast cancer. Abbreviation: BC, breast cancer; GWAS, genome-wide association studies. 
Among the set of well-established common susceptibility genes are variants in intron 2 of the $F G F R 2$ gene, ${ }^{24}$ which, among the common variants, are likely to make one of the larger contributions to relative risk, at least for postmenopausal disease. Easton et $\mathrm{al}^{25}$ found that the rs2981582 SNP (allele frequency 0.38 ) contributes odds ratios of 1.23 and 1.63 for heterozygote and homozygote genotypes, respectively. The FGFR2 gene encodes a fibroblast growth factor (FGF) receptor. FGFs and their corresponding receptors are involved in regulation of the proliferation, survival, migration, and differentiation of cells. The considerable importance of FGF signaling in a range of tumor types is now becoming recognized. ${ }^{26}$ SNPs within intron 2 are involved in FGFR2 upregulation, and aberrant signaling activation induces proliferation and survival of tumor cells. ${ }^{27}$ The identification of this gene, which was unanticipated as a cancer gene, has prompted research into related genes and their potential roles in cancer. Other FGFs (eg, FGF-8) appear to be involved in breast cancer cell growth through stimulation of cell cycle and prevention of cell death. ${ }^{28}$

Other low-penetrance variants that have been identified through GWAS include CASP8 (caspase 8), which encodes an apoptotic enzyme. ${ }^{29}$ The variant rs 1045485 is protective, contributing odds ratios of 0.89 and 0.74 for heterozygotes and rare homozygotes, respectively. Recently, variants in CASP8 have been shown to alter risks (in a protective direction) in individuals with a family history of breast cancer. $^{30}$

Breast tumors are classified according to whether they have receptor proteins that bind to estrogen and progesterone. Such cells are termed ER+ and PR+ and require estrogen and progesterone to grow. Conversely, ER- and PR- tumors lack the protein that allows the hormones to bind. Tumor classifications influence the choice of treatment regimes for the patient. A further classification arises through tumors that overexpress the human epidermal growth factor receptor 2 (HER2) gene, which are termed HER2+ (conversely, HER2-). The triple-negative subtypes are ER-, PR-, and HER2- and are characterized by aggressive tumors and reduced range of effective treatment options. Several common gene variants are more strongly associated with specific cancer subtypes. These include the TOX3 gene, formerly called TNRC9 in which variant rs3803662 contributes a 1.64-fold homozygote risk, specifically in ER+ cancer. ${ }^{31}$ This gene encodes a high-mobility group chromatin-associated protein and increased expression is implicated in bone metastasis. ${ }^{32}$ Fine mapping has shown that hypothesized susceptibility variants lie in an intergenic region consistent with a gene regulatory function. ${ }^{33}$ These authors note there remains uncertainty as to whether the causal variant is actually involved in the regulation of the nearby retinoblastoma-like gene 2 ( $R B L 2)$ gene, which is involved in cell cycle regulation, given gene expression evidence.

The mitogen-activate protein kinase (MAP3K1) breast cancer gene ${ }^{25}$ is a member of the Ras/Raf/MEK/ERK signaling pathway (as is FGFR2) and is involved in regulating transcription of a number of cancer genes. MAP $3 K 1$ has been found to be more strongly associated with ER+ and $\mathrm{PR}+$ tumors than ER-/PR- subtypes. There is also a stronger association with HER2+ tumors. ${ }^{34}$

The $L S P 1$ gene was identified as a breast cancer susceptibility locus by Easton et al, ${ }^{25}$ who identified an SNP within intron 10 as the most strongly associated. LSP1 encodes lymphocyte-specific protein 1, which is an F-actin binding cytoskeletal protein. The same study also identified a breast cancer variant in the 8q24 region containing no known genes. This region is also associated with prostate cancer..$^{35}$

Stacey et $\mathrm{a}^{31}$ identified a SNP on $2 \mathrm{q} 35$, a region with no known genes, as associated with breast cancer in Icelandic patients with ER+ breast cancer. Milne et $a l^{36}$ also found an association with ER- disease, although there was a stronger signal for ER+. Other breast cancer associations include signals on $3 \mathrm{p} 24$, potentially relating to the genes SLC4A7 or NEK10, and on 17q22, perhaps related to COX11. These SNPs contribute odds ratios of 1.11 and 0.97 for heterozygote and homozygote genotypes, respectively. ${ }^{37}$ Additionally, a common variant close to MRPS30 on $5 \mathrm{p} 12$ was found to confer higher risk of ER+ disease. ${ }^{38}$ Turnbull et $\mathrm{al}^{39}$ described five new associations on chromosomes 9 , 10 (three regions), and 11 . Two further signals reported by Thomas et $\mathrm{al}^{40}$ include a SNP in the pericentromeric part of chromosome 1, within a region containing $\mathrm{NOTCH} 2$ and $F C G R 1 B$, and a signal associated with another double-strand break repair gene (RAD51L1) on 14q24.1. There is evidence that the chromosome 1 locus is more strongly associated with ER+ disease.

Considerable additional follow-up investigation will be required to establish the relationships between many of the SNPs and the actual causal variant(s) and to further elucidate the role in disease for many of these common genes.

\section{The genetic basis of breast cancer subphenotypes}

Analysis of breast cancer as a single phenotype is becoming less typical as genetic differences between disease subtypes 
are more clearly established. Increased power to detect genetic variants is expected using patients belonging to genetically more homogeneous subgroups, rather than analyzing more heterogeneous groupings. There is evidence that many breast cancer GWAS studies have been enriched with ER+ cases because ER positivity is found in a higher proportion of the later-onset (usually postmenopausal) cases used in most of these studies. For this reason, ER+ disease is better characterized genetically than ER- disease. For example, Stacey et $\mathrm{al}^{38}$ identified two SNPs on chromosome 5p12 that confer risk preferentially for ER+ tumors. Garcia-Closas et $\mathrm{al}^{41}$ showed that variants in FGFR2 are more strongly related to ER+ than ER- (and also more strongly associated with $\mathrm{PR}+$, low tumor grade, and lymph node-positive tumors). The breast cancer association in the $8 \mathrm{q} 24$ region is significantly stronger for $\mathrm{ER}+, \mathrm{PR}+$, and low-grade tumors. Reeves et $\mathrm{al}^{42}$ examined risk odds ratios for low-penetrance breast cancer genes in a sample of more than 10,000 cases and controls in relation to ER+ and ER- classification, for bilateral and unilateral disease, and for lobular versus ductal tumors. They noted higher odds ratios for ER+ disease for FGFR2 and TCNR9, compared with ER- disease, greater association with bilateral, compared with unilateral, and for lobular disease compared with ductal disease in the 2 q region. Using a polygenic risk score, based on seven breast cancer SNPs, the estimated cumulative incidence in the top fifth of the score distribution for ER+ disease is $7.4 \%$ compared with only $1.4 \%$ for ER- disease. Since the polygenic risk score is substantially more strongly predictive for ER+ disease, there is a strong case for more thorough evaluation of the genetic basis of the ER-subtype.

Triple-negative breast cancers are associated with poor prognosis due to aggressive tumor behavior and poor response to chemotherapy. ${ }^{43}$ After screening 2301 triplenegative cases and 3949 controls, Antoniou et al ${ }^{44}$ identified five SNPs on 19p13 that modify risk in BRCA1 mutation carriers and are specifically associated with triple-negative breast cancer. Additional phenotypic subtypes which are currently being interrogated genetically include differences in susceptibility variants between racial groups and in response to treatment and prognosis.

\section{Genetic risk factors for breast cancer: clinical applications}

Mutations in the $B R C A 1$ and $B R C A 2$ genes are rare but underlie severe and early-onset forms of the disease. Screening for mutations in women with a strong family history, usually linked to BRCA mutations, determines individual risks for this early-onset form of disease. However, most patients $(\sim 95 \%)$ do not show clear-cut family histories of early- or later-onset disease. The role of more common breast cancer variation in risk prediction is far less well established. Pharoah et $\mathrm{al}^{45}$ determined a multiplicative model using the 12 most significant common variants to define individual relative risks in the range 0.4 -fold to fourfold compared with the general population. Given that there is a $12 \%$ population lifetime risk, deleterious common mutations contribute a $24 \%-36 \%$ lifetime risk, which may be high enough to instigate earlier and more intensive screening for common genetic forms of the disease. Gulcher and Stefansson ${ }^{46}$ point out that some women classified as at average risk would be reclassified as at higher risk based on their profile of common breast cancer variation. Similarly, some women might be reclassified as having lower-than-average risk based on their common gene profile. Risk estimates might be more reliably determined by multiplying risks from the genetic profiles with independent risks from conventional measures, such as family history, age at menarche, and pregnancy history. Successful application of common breast cancer gene profiles in clinical practice would have potential benefits by facilitating earlier diagnosis, reduced costs, less intensive therapeutic intervention, and disease management in the longer term.

As understanding of the genetic basis of breast cancer increases, further refinement in genetic risk models can be expected. The different genetic basis of tumor subtypes is a clear example of where refinement might take place as genetic profiles become predictive of tumor characteristics. At this stage, it is already well established that women with, or at higher risk for, $\mathrm{ER}+$ cancer are a good candidates for treatment with tamoxifen or raloxifene that specifically targets ER+ disease.

\section{Finding the missing heritability}

The breast cancer genes identified thus far explain only about $30 \%$ of the heritability, which is the proportion of the phenotypic variance that can be attributed to genetic variation. There are several possible sources for the missing genes, and this is a subject of intense argument and ongoing research.

\section{Undetected common variation}

GWAS using SNPs target only high-frequency alleles, and risk alleles found through these methods all have frequencies well in excess of $0.05 .^{22}$ Even within this common allele 'window', the SNP panels provide incomplete genome coverage, due in part to technical limitations of the genotyping platforms, but mainly due to cost, which places reliance on tagging SNPs (using a SNP in linkage disequilibrium with many others to 
represent or tag a specific haplotype). Such an approach is cost effective but loses information. ${ }^{47}$ Furthermore, these platforms are relatively enriched for nonsynonymous coding SNPs (cSNPs), so the coverage of synonymous cSNPs and noncoding SNPs is incomplete. Given that common disease variants include a higher proportion of regulatory SNPs, which lie outside coding regions, it is likely that important common variation has been missed by the GWAS undertaken thus far. Because effect sizes of common variants are low, very large samples of cases and controls are required for effective GWAS. Many as yet undetected common variants will have increasingly small effects on risk as variants with larger effect sizes will have already been detected through the completed GWAS. The largest study to date of common variation underlying a complex trait is the analysis of the genetic basis of height. Allen et $\mathrm{al}^{48}$ tested data from 183,727 individuals and identified hundreds of common genetic variants in at least 180 loci that account for $\sim 10 \%$ of the phenotypic variation in height. They estimated that as yet unidentified common variation (with similar effect sizes to those already found) will eventually account for $\sim 20 \%$ of the heritable variation, but detecting these would require a sample size of 500,000 individuals. Importantly, they concluded that many genetic loci underlying variation in height show allelic heterogeneity suggesting that as yet unidentified causal variants will map to the loci already identified in GWAS. These missing variants are likely to span the allele frequency spectrum, including rare variants with higher penetrance, but the remaining low-penetrance variants can only be detected by ever-larger GWAS.

\section{Structural variation}

Structural variation, such as copy number variants (CNVs), which are not well tagged by SNPs in current arrays, may be a source of missing heritability in breast cancer. There is evidence that at least the common CNVs are in strong linkage disequilibrium with common SNPs genotyped in GWAS and hence may be adequately 'tagged' by existing panels. ${ }^{49}$ Significant associations with rare CNVs (frequency range $0.2 \%-1 \%$ ) have been identified for a number of neuropsychiatric traits, such as autism, epilepsy, and mental retardation, ${ }^{50}$ although no CNVs have been convincingly associated with cancer phenotypes thus far. ${ }^{49}$

\section{Gene-gene and gene-environment interaction}

Other possibilities include interaction effects between genes and between genes and environment. Exploring such scenarios presents analytical challenges and there is relatively limited evidence for an important role for interaction thus far. Ritchie et $\mathrm{al}^{51}$ modeled data for $10 \mathrm{SNPs}$ in the genes COMT, CYP1A1, CYP1B1, GSTM1, and GSTT1. They identified an interaction between all the genes that were significantly associated with increased risk for sporadic breast cancer. Briollais et al ${ }^{52}$ also identified SNP-SNP interactions associated with breast cancer, including an interaction between XPD and IL10 genes as the most significant two-way interaction. Travis et $\mathrm{al}^{53}$ examined the relationship between environmental variables, such as reproductive, behavioral, and anthropometric factors, with low-penetrance breast cancer genes. After allowing for multiple testing, they observed no evidence for increased breast cancer risk arising through gene-environment interaction in their sample of 7610 women. Because of the potentially huge number of statistical tests in such comparisons, obtaining a large enough sample to have power to demonstrate an effect can be difficult. Furthermore, confirmatory studies, along with functional analyses of the biological pathways involved, are essential to fully comprehend the importance of putative gene-gene and gene-environment interactions. ${ }^{54}$ Moore et $\mathrm{al}^{55}$ argued that the information gleaned from GWAS data collected thus far has been limited by failure to integrate existing knowledge about disease pathology: the 'single SNP' analysis approach ignores the genomic and environmental context. They recommend enhanced bioinformatic approaches to develop a holistic approach that recognizes the full complexity of gene-phenotype, gene-gene, and geneenvironment interactions.

\section{Undetected rare variation}

Searching for rarer variants with larger effect sizes is likely to be a successful strategy for identifying more of the missing heritability. Rare variants have not been screened by GWAS, so this source of novel genetic variation is largely unexplored. Rare variants may contribute odds ratios in the range $2-5$, compared with common variants that typically have odds ratios $<1.5 .^{56}$ Targets for ongoing and future studies include low-frequency variants with minor allele frequencies in the $0.3 \%-5 \%$ range. Studies exploiting next-generation sequencing include Johansen et al, ${ }^{57}$ who tested association with high triglyceride levels and resequenced loci previously identified as containing common variation. They found approximately twice as many rare coding genetic variants associated with high triglycerides located within the same genes. Determining the extent to which low-frequency and rare causal variants are colocated within breast cancer loci already identified in GWA studies 
depends on future sequencing efforts targeting these wellestablished genes. There is clearly a strong case to examine susceptibility variants over the full allele frequency range. ${ }^{56}$ Next-generation sequencing for the analysis of breast cancer exomes (the $\sim 30 \mathrm{Mb}$ of sequence within protein-coding exons) of patients with early onset and a strong family history, which are negative for known highly penetrant rare mutations (BRCA1, BRCA2, and TP53), are likely to be informative. Using exome sequencing, the full complement of, for example, SNPs and insertion-deletion polymorphisms can be characterized in every sample. Support for this strategy comes from the identification of rare highly penetrant mutations in the $R A D 51 C$ gene. ${ }^{16}$ For less completely penetrant variants, there are, however, many more difficulties in assessing the significance of the variation identified. The 1000 genomes project ${ }^{23}$ provides reference sequence for studying relationships between phenotype and genotype. The pilot phase describes exon-targeted sequencing of 697 individuals and whole genome sequencing of 179 individuals. The study determined the location and frequency of 15 million SNPs, 1 million insertion/deletion polymorphisms, and 20,000 structural variants. Comparing the pattern of variation identified in disease samples with this catalog of 'normal' variation is a crucial step in the process of determining the disease significance of any variants found.

Scale-up to sequence exomes of much larger samples of cases to investigate variants with intermediate and lower penetrance has not been undertaken thus far. Large samples will be required, and genetic heterogeneity, combined with the huge volume of (mostly unimportant) variation uncovered, poses extreme challenges for data interpretation even given knowledge about 'background' variation provided by the 1000 genomes project. In these cases, assessment of potential functional roles for variants found requires integration of information on gene expression profiles and other sources of transcriptome data and implementation of bioinformatic approaches to predict functional effects. Exome sequencing has its limitations. Apart from the fact that only exons are screened and that much important variation is known to reside outside these regions, there is limited information on structural variation, such as CNVs. Whole genome sequencing will generate a complete catalog of the variation, but many issues concerning the management, analysis, and interpretation of the huge volumes of data generated are not yet resolved.

\section{Conclusion}

In recent years, $\sim 30$ genes and gene regions have been confirmed as containing variants underlying susceptibility to breast cancer. The majority of recent discoveries have been low-penetrance common variants identified through GWAS with SNPs. Disease risks associated with these SNPs are low, typically much $<1.5$-fold. In many cases, the causal variant is unknown, and the associated marker is only in linkage disequilibrium with the actual site. In the majority of the cases, the role of these variants in causing disease is also unknown, but ongoing study is revealing novel insights into breast cancer biology. Areas of intensive research include investigation of the genetic basis of disease subtypes, for which there appear to be marked genetic differences, the impact of genetic variation on prognosis and on response to treatment. Despite the huge amount of work undertaken thus far, $\sim 70 \%$ of the disease heritability remains unexplained. The common, low-penetrance variants identified through GWAS have contributed only a small proportion of this missing heritability. Aside from rare variants in the BRCAI and $B R C A 2$ genes and a small number of other rare genes that show approximately Mendelian patterns of inheritance, the majority of breast cancer genes found contribute little toward the prediction of individual disease risk. A thorough understanding of the biological role of the variation detected is some way off, and much more detailed functional and bioinformatic analysis is required for further progress. In the meantime, analysis of breast cancer exomes to identify SNPs and insertion-deletion polymorphisms will provide important insights by providing the first opportunity to examine rarer forms of variation in coding regions. This strategy will be effective for variants with higher penetrance, but where penetrance is toward the lower end of the spectrum, interpretation of the roles of numerous rarer variants will present new challenges for bioinformatic and functional assays. Once these problems are resolved, exome and whole genome sequencing strategies are likely to offer the best opportunity to identify additional breast cancer genetic risk factors. The identification of these genes is the crucial first step in fully comprehending the biology of disease and moving toward individualized treatments.

\section{Disclosure}

The authors report no conflicts of interest in this work.

\section{References}

1. Collaborative Group on Hormonal Factors in Breast Cancer. Familial breast cancer: collaborative reanalysis of individual data from 52 epidemiological studies including 58,209 women with breast cancer and 101,986 women without the disease. Lancet. 2001;358(9291): 1389-1399.

2. Hall JM, Lee MK, Newman B, et al. Linkage of early-onset familial breast cancer to chromosome 17q21. Science. 1990;250(4988):1684-1689. 
3. Wooster R, Neuhausen SL, Mangion J, et al. Localization of a breast cancer susceptibility gene, BRCA2, to chromosome 13q12-13. Science. 1994;265(5181):2088-2090.

4. Stratton MR, Rahman N. The emerging landscape of breast cancer susceptibility. Nat Genet. 2008;40(1):17-22.

5. Peto J, Collins N, Barfoot R, et al. Prevalence of BRCA1 and BRCA2 gene mutations in patients with early-onset breast cancer. J Natl Cancer Inst. 1999;91(11):943-949.

6. Bodmer W, Tomlinson I. Rare genetic variants and the risk of cancer. Curr Opin Genet Dev. 2010;20(3):262-267.

7. Venkitaraman AR. Cancer susceptibility and the functions of BRCA1 and BRCA2. Cell. 2002;108(2):171-182.

8. Yun MH, Hiom K. CtIP-BRCA1 modulates the choice of DNA doublestrand-break repair pathway throughout the cell cycle. Nature. 2009; 459(7245):460-463.

9. Huen MS, Sy SM, Chen J. BRCA1 and its toolbox for the maintenance of genome integrity. Nat Rev Mol Cell Biol. 2010;11(2):138-148.

10. Jensen RB, Carreira A, Kowalczykowski SC. Purified human BRCA2 stimulates RAD51-mediated recombination. Nature. 2010; 467(7316):678-683.

11. Børresen AL, Andersen TI, Garber J, et al. Screening for germ line TP53 mutations in breast cancer patients. Cancer Res. 1992; 52(11):3234-3236.

12. Nelen MR, Padberg GW, Peeters EA, et al. Localization of the gene for Cowden disease to chromosome 10q22-23. Nat Genet. 1996; 13(1):114-116.

13. Boardman LA, Thibodeau SN, Schaid DJ, et al. Increased risk for cancer in patients with the Peutz-Jeghers syndrome. Ann Intern Med. 1998; 128(11):896-899.

14. Antoniou AC, Easton DF. Models of genetic susceptibility to breast cancer. Oncogene. 2006;25(43):5898-5905.

15. Berx G, Van Roy F. The E-cadherin/catenin complex: an important gatekeeper in breast cancer tumorigenesis and malignant progression. Breast Cancer Res. 2001;3(5):289-293.

16. Meindl A, Hellebrand H, Wiek C, et al. Germline mutations in breast and ovarian cancer pedigrees establish RAD51C as a human cancer susceptibility gene. Nat Genet. 2010;42(5):410-414.

17. Thompson D, Duedal S, Kirner J, et al. Cancer risks and mortality in heterozygous ATM mutation carriers. J Natl Cancer Inst. 2005; 97(11):813-822.

18. Rahman N, Seal S, Thompson D, et al. PALB2, which encodes a BRCA2-interacting protein, is a breast cancer susceptibility gene. Nat Genet. 2007;39(2):165-167.

19. Robson M. CHEK2, breast cancer, and the understanding of clinical utility. Clin Genet. 2010;78(1):8-10.

20. Dickson SP, Wang K, Krantz I, Hakonarson H, Goldstein DB. Rare variants create synthetic genome-wide associations. PLoS Biol. 2010; 8(1):e1000294.

21. Orozco G, Barrett JC, Zeggini E. Synthetic associations in the context of genome-wide association scan signals. Hum Mol Genet. 2010; 19(R2):R137-R144.

22. Hindorff LA, Sethupathy P, Junkins HA, et al. Potential etiologic and functional implications of genome-wide association loci for human diseases and traits. Proc Natl Acad Sci U S A. 2009; 106(23):9362-9367.

23. 1000 Genomes Project Consortium, Durbin RM, Abecasis GR, Altshuler DL, et al. A map of human genome variation from populationscale sequencing. Nature. 2010;467(7319):1061-1073.

24. Hunter DJ, Kraft P, Jacobs KB, et al. A genome-wide association study identifies alleles in FGFR2 associated with risk of sporadic postmenopausal breast cancer. Nat Genet. 2007;39(7):870-874.

25. Easton DF, Pooley KA, Dunning AM, et al. Genome-wide association study identifies novel breast cancer susceptibility loci. Nature. 2007; 447(7148):1087-1093.

26. Turner N, Grose R. Fibroblast growth factor signalling: from development to cancer. Nat Rev Cancer. 2010;10(2):116-129.
27. Katoh Y, Katoh M. FGFR2-related pathogenesis and FGFR2-targeted therapeutics (Review). Int J Mol Med. 2009;23(3):307-311.

28. Nilsson EM, Brokken LJ, Härkönen PL. Fibroblast growth factor 8 increases breast cancer cell growth by promoting cell cycle progression and by protecting against cell death. Exp Cell Res. 2010; 316(5):800-812.

29. Cox A, Dunning AM, Garcia-Closas M, et al. A common coding variant in CASP8 is associated with breast cancer risk. Nat Genet. 2007;39(3):352-358.

30. Latif A, Hadfield KD, Roberts SA, et al. Breast cancer susceptibility variants alter risks in familial disease. J Med Genet. 2010; 47(2):126-131.

31. Stacey SN, Manolescu A, Sulem P, et al. Common variants on chromosomes $2 \mathrm{q} 35$ and $16 \mathrm{q} 12$ confer susceptibility to estrogen receptorpositive breast cancer. Nat Genet. 2007;39(7):865-869.

32. Smid M, Wang Y, Klijn JG, et al. Genes associated with breast cancer metastatic to bone. J Clin Oncol. 2006;24(15):2261-2267.

33. Udler MS, Ahmed S, Healey CS, et al. Fine scale mapping of the breast cancer 16q12 locus. Hum Mol Genet. 2010;19(12):2507-2515.

34. Rebbeck TR, DeMichele A, Tran TV, et al. Hormone-dependent effects of FGFR2 and MAP3K1 in breast cancer susceptibility in a population-based sample of post-menopausal African-American and European-American women. Carcinogenesis. 2009;30(2):269-274.

35. Amundadottir LT, Sulem P, Gudmundsson J, et al. A common variant associated with prostate cancer in European and African populations. Nat Genet. 2006;38(6):652-658.

36. Milne RL, Benítez J, Nevanlinna H, et al. Risk of estrogen receptor-positive and -negative breast cancer and single-nucleotide polymorphism 2q35-rs13387042. J Natl Cancer Inst. 2009;101(4):1012-1018.

37. Ahmed S, Thomas G, Ghoussaini M, et al. Newly discovered breast cancer susceptibility loci on 3p24 and 17q23.2. Nat Genet. 2009; 41(5):585-590.

38. Stacey SN, Manolescu A, Sulem P, et al. Common variants on chromosome $5 \mathrm{p} 12$ confer susceptibility to estrogen receptor-positive breast cancer. Nat Genet. 2008;40(6):703-706.

39. Turnbull C, Ahmed S, Morrison J, et al. Genome-wide association study identifies five new breast cancer susceptibility loci. Nat Genet. 2010; 42(6):504-509

40. Thomas G, Jacobs KB, Kraft P, et al. A multistage genome-wide association study in breast cancer identifies two new risk alleles at 1 p11.2 and 14q24.1 (RAD51L1). Nat Genet. 2009;41(5):579-584.

41. Garcia-Closas M, Hall P, Nevanlinna H, et al. Heterogeneity of breast cancer associations with five susceptibility loci by clinical and pathological characteristics. PLoS Genet. 2008;4(4):e1000054.

42. Reeves GK, Travis RC, Green J, et al. Incidence of breast cancer and its subtypes in relation to individual and multiple low-penetrance genetic susceptibility loci. JAMA. 2010;304(4):426-434.

43. Podo F, Buydens LM, Degani H, et al. Triple-negative breast cancer: present challenges and new perspectives. Mol Oncol. 2010; 4(3):209-229.

44. Antoniou AC, Wang X, Fredericksen ZS, et al. A locus on 19 p13 modifies risk of breast cancer in BRCA1 mutation carriers and is associated with hormone receptor-negative breast cancer in the general population. Nat Genet. 2010;42(10):885-892.

45. Pharoah PD, Antoniou AC, Easton DF, Ponder BA. Polygenes, risk prediction, and targeted prevention of breast cancer. $N$ Engl J Med. 2008;358(26):2796-2803.

46. Gulcher J, Stefansson K. Genetic risk information for common diseases may indeed be already useful for prevention and early detection. Eur J Clin Invest. 2010;40(1):56-63.

47. Zhang W, Collins A, Morton NE. Does haplotype diversity predict power for association mapping of disease susceptibility? Hum Genet. 2004;115(2):157-164.

48. Allen HL, Estrada K, Lettre G, et al. Hundreds of variants clustered in genomic loci and biological pathways affect human height. Nature. 2010;467(7317):832-838 
49. Galvan A, Ioannidis JP, Dragani TA. Beyond genome-wide association studies: genetic heterogeneity and individual predisposition to cancer. Trends Genet. 2009;26(3):132-141.

50. Williams HJ, Owen MJ, O’Donovan MC. Schizophrenia genetics: new insights from new approaches. Br Med Bull. 2009;91:61-74.

51. Ritchie MD, Hahn LW, Roodi N, et al. Multifactor-dimensionality reduction reveals high-order interactions among estrogen-metabolism genes in sporadic breast cancer. Am J Hum Genet. 2001;69(1):138-147.

52. Briollais L, Wang Y, Rajendram I, et al. Methodological issues in detecting gene-gene interactions in breast cancer susceptibility: a population-based study in Ontario. BMC Med. 2007;5:22.

53. Travis RC, Reeves GK, Green J, et al. Gene-environment interactions in 7610 women with breast cancer: prospective evidence from the Million Women Study. Lancet. 2010;375(9732):2143-2151.
54. Spurdle AB, Chang JH, Byrnes GB, et al. A systematic approach to analysing gene-gene interactions: polymorphisms at the microsomal epoxide hydrolase $E P H X$ and glutathione $S$-transferase GSTM1, GSTT1, and GSTP1 loci and breast cancer risk. Cancer Epidemiol Biomarkers Prev. 2007;16(4):769-774.

55. Moore JH, Asselbergs FW, Williams SM. Bioinformatics challenges for genome-wide association studies. Bioinformatics. 2010; 26(4):445-455.

56. Gloyn AL, McCarthy MI. Variation across the allele frequency spectrum. Nat Genet. 2010;42(8):648-650.

57. Johansen CT, Wang J, Lanktree MB, et al. Excess of rare variants in genes identified by genome-wide association study of hypertriglyceridemia. Nat Genet. 2010;42(8):684-687.

\section{Publish your work in this journal}

The Application of Clinical Genetics is an international, peer-reviewed open access journal that welcomes laboratory and clinical findings in the field of human genetics. Specific topics include: Population genetics; Functional genetics; Natural history of genetic disease; Managemen of genetic disease; Mechanisms of genetic disease; Counselling and

\section{Dovepress}

ethical issues; Animal models; Pharmacogenetics; Prenatal diagnosis; Dysmorphology. The manuscript management system is completely online and includes a very quick and fair peer-review system, which is all easy to use. Visit http://www.dovepress.com/testimonials.php to read real quotes from published authors.

Submit your manuscript here: http://www.dovepress.com/the-application-of-clinical-genetics-journal 